\title{
Length Scale Analysis of Surface Energy Fluxes Derived from Remote Sensing
}

\author{
NATHANiEl A. BRUNSELL* \\ Department of Plants, Soils, and Biometeorology, Utah State University, Logan, Utah \\ ROBERT R. GILLIES \\ Department of Plants, Soils, and Biometeorology, and Department of Aquatic, Watershed and Earth Resources, Utah State University, \\ Logan, Utah
}

(Manuscript received 7 August 2002, in final form 6 June 2003)

\begin{abstract}
Wavelet multiresolution analysis was used to examine the variation in dominant length scales determined from remotely sensed airborne- and satellite-derived surface energy flux data. The wavelet cospectra are computed between surface radiometric temperature, fractional vegetation, and derived energy fluxes at airborne $(12 \mathrm{~m})$ and Advanced Very High Resolution Radiometer (AVHRR) (1000 m) resolutions. Length scale analysis of highresolution data shows that small-scale variability in temperature dominates over other effects. Analysis of coarseresolution data shows that small-scale variations in vegetation are important, although the large-scale variation in radiometric temperature dominates the derived fluxes. This is determined to be a result of the fact that, at smaller scales, the incoming solar radiation effect is muted by the small-scale variability in vegetation, temperature, and albedo, whereas at coarser scales, the large-scale effect of incoming radiation on temperature dominates over the smaller-scale features in surface variability.
\end{abstract}

\section{Introduction}

An understanding of the interaction between surface spatial variability in vegetation (rather than variability in vegetation types) and the composite surface radiometric temperature and the resultant turbulent fluxes is important for the ability to compute large-scale estimations of the surface energy balance with remote sensing. A problem in land-atmosphere-interactions research is that the processes that govern the transfer of mass, energy, and momentum across the land-atmosphere interface are nonlinear, because of the interdependence of the dominant variables and parameters. Specifically, this amounts to the fact that the average value of a flux is not necessarily a function of the average value of the controlling variables and parameters. It is therefore not enough to know the spatial distribution of the controlling variables; it is necessary to understand how these distributions are altered with change in scale.

Insight into this issue can potentially be gained by the collection of remotely sensed data at different res-

\footnotetext{
* Current affilication: Civil and Environmental Engineering, Duke University, Durham, North Carolina.
}

Corresponding author address: Dr. Nathaniel A. Brunsell, Civil and Environmental Engineering, Duke University, Hudson Hall, Box 90287, Durham, NC 27708-0287.

E-mail: brunsell@duke.edu olutions. The sensor aboard a satellite or aircraft averages the radiance emitted and reflected by the surface as transmitted through the atmosphere. This provides information concerning the radiative balance of the surface at the resolution of the sensor, assuming the energy balance is closed at all spatial resolutions.

There have been a number of extensive field campaigns [e.g., the First International Satellite Land Surface Climatology Project (ISLSCP) Field Experiment (FIFE), and the Boreal Ecosystem-Atmosphere Study (BOREAS), etc.] that have attempted to collect field data simultaneously at a variety of spatial scales. However, many of these field projects have, for one reason or another, failed to collect multiple resolutions of remotely sensed data by both aircraft and satellite on the same day. A partial exception to this is the Southern Great Plains 1997 (SGP97) Hydrology Experiment in Oklahoma in the summer of 1997.

SGP97 provided an excellent opportunity to examine scale issues involved with remotely sensed data, because of the wide variety of remotely sensed and surface measurements collected within a relatively short time span (approximately 1 month) (Kustas et al. 2001). The airborne remotely sensed data used in this study includes those collected by the Thematic Mapper Simulator (TMS) and the Thermal Infrared Multispectral Scanner (TIMS). Both of these datasets have a $12-\mathrm{m}$ pixel resolution. Data were collected for 2 July 1997 over the 
El Reno, Oklahoma, study area (French et al. 2000). In terms of surface measurements, four eddy-covariance flux towers were incorporated into the analysis and provided a variety of meteorological data. In addition, satellite data from the 2 July 1997 Advanced Very High Resolution Radiometer (AVHRR) overpass were available and cloud free over the study area.

There are several parts to this paper and it is beneficial to give a short overview here. The surface energy fluxes are computed for the TMS/TIMS and AVHRR datasets via the "triangle method" of Gillies et al. (1997). The length scales of each flux are subsequently computed for both resolutions in conjunction with the cospectra between the derived fluxes and both the radiometric surface temperature and the fractional vegetation. For the purposes of this paper, the terms temperature and radiometric surface temperature are used interchangeably and refer to the temperature measured by a radiometer and corrected for atmospheric and surface emissivity effects.

The SGP97 dataset provides an opportunity to examine how the variability across different spatial resolutions in input data translates into variation across scales in modeled output. This issue is of prime importance to the remote sensing community involved in the modeling of land-atmosphere interactions. It is often assumed that the variability observed in the remote sensing data is "true" and, more importantly, that this variability is the dominant variability that exists within the environment from which the data were collected. Therefore, the application of models to derive the various components of the surface energy balance are applied to the remote sensing input, regardless of the initial resolution of the data. This inherently assumes that the model formulation is valid for the complete range of scales that it is possible to obtain with remote sensing. However, it is rarely possible to examine whether or not the spatial variability observed at one scale manifests itself in similar estimates at another scale. Through the use of the same model at different resolutions at the same time, this paper examines some of these issues.

Wavelet transforms provide a technique that is perfectly suited to investigating these issues. The insensitivity of the wavelet transform to nonstationarities in the data is beneficial over more standard techniques (e.g., Fourier-based techniques, semivariograms, etc.), particularly when examining nonlinear interactions such as those associated with estimating surface energy fluxes from remote sensing data.

An understanding of the variation of remotely sensed input fields (e.g., radiometric temperature and fractional vegetation) and the effect on surface fluxes is important for a variety of reasons. One reason is the issue of assimilation of remotely sensed data into mesoscale and other meteorological and climatological models, both in an investigative capacity as well as on an operational basis. Moreover, this information could increase the confidence in hydrological modeling of the large-scale water balance. Of direct importance here is the computation of spatially distributed evaporation over model scales at an hourly time step [e.g., with the use of the Geostationary Operational Environmental Satellite (GOES) (Diak 1990)]. However, before this is feasible, an understanding between the interaction of remotely sensed data and derived surface energy fluxes must be developed.

\section{Methodology}

a. Remotely sensed data corrections

1) NDVI

The Normalized Difference Vegetation Index (NDVI) was calculated for the TMS, TM, and AVHRR datasets. NDVI is defined in terms of the surface reflectance:

$$
\text { NDVI }=\frac{\rho_{2}-\rho_{1}}{\rho_{2}+\rho_{1}},
$$

where $\rho_{1}$ is the surface reflectance in the red band and $\rho_{2}$ is the near-infrared reflectance.

The fractional vegetation is defined as (Gillies et al. 1997)

$$
\mathrm{Fr}=\left(\frac{\mathrm{NDVI}-\mathrm{NDVI}_{\text {soil }}}{\mathrm{NDVI}_{\mathrm{veg}}-\mathrm{NDVI}_{\mathrm{soil}}}\right)^{2}
$$

where $\mathrm{NDVI}_{\text {soil }}$ and $\mathrm{NDVI}_{\mathrm{veg}}$ are the bare soil and fully vegetated NDVI values, respectively. These values are taken from the image under analysis as the minimum value corresponding to land surface pixels (i.e., excluding water and cloud pixels) for the soil NDVI and the maximum value within the image for the vegetation NDVI. This necessitates the assumption that the image contains both bare soil and fully vegetated pixels. This equation has been empirically derived and discussed by several authors, including Choudhury et al. (1994), Gillies et al. (1997), and Carlson and Ripley (1997).

Surface reflectance values were obtained via an atmospheric correction with the radiative transfer model MODTRAN3 (Kniezys et al. 1996) for the TMS and AVHRR data. This correction algorithm relates surface reflectance to apparent (at sensor) radiance and sensor scan angle. This is done by initializing MODTRAN with a local radiosonde profile, then iterating the model over the range of scan angles, and generating a least squares regression to calculate surface reflectance. This correction incorporates the effects of look angle, since these effects are not symetric around nadir in the visible and near-infrared regions of the electromagnetic spectrum.

\section{2) RADIOMETRIC TEMPERATURE}

The six bands of TIMS and band 4 of AVHRR were corrected to radiometric surface temperature via Brunsell and Gillies (2002a), which incorporates an esti- 

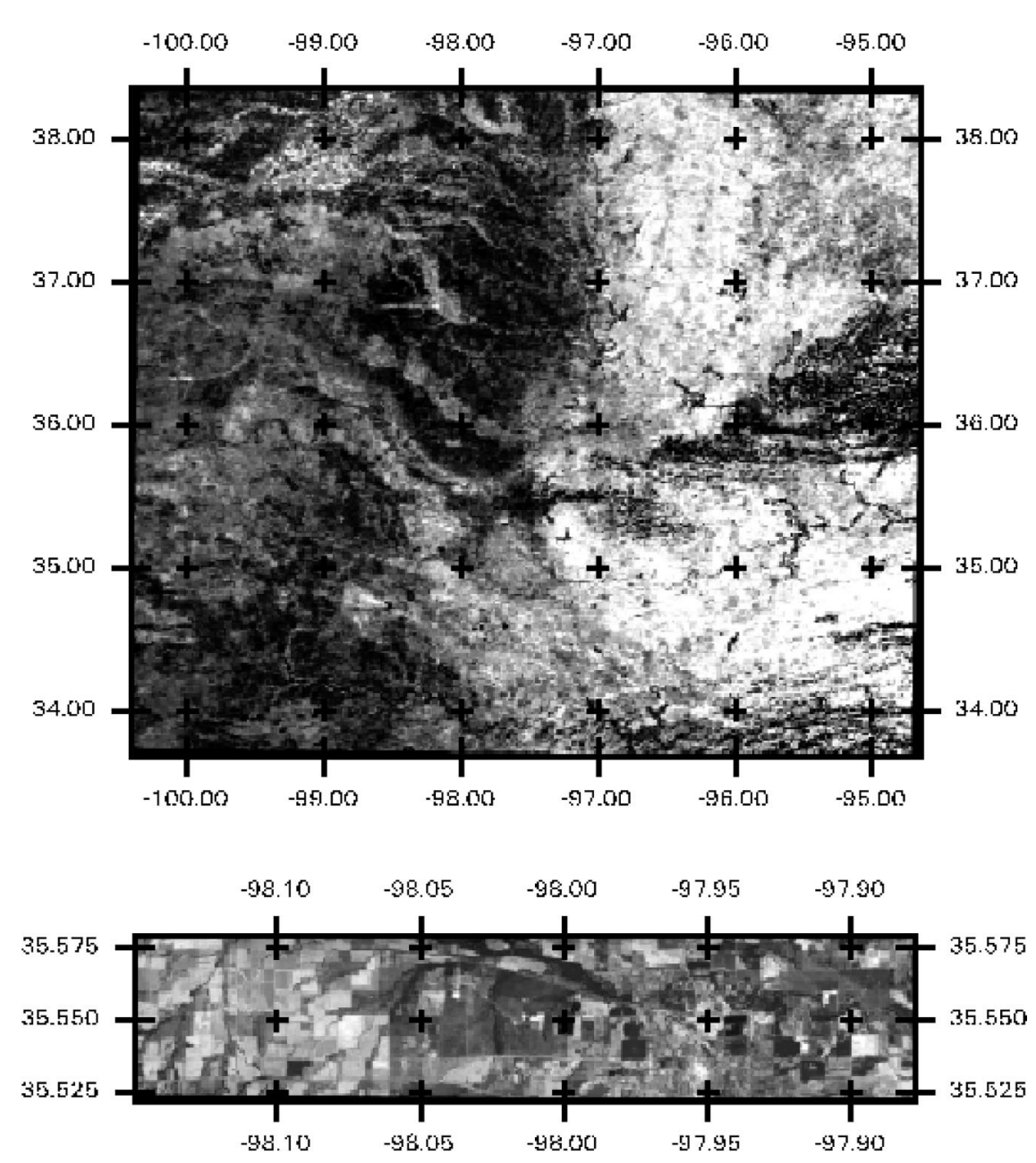

FIG. 1. The extent of the (top) AVHRR (approximately 1000-m resolution and $512 \times 512 \mathrm{~km}^{2}$ extent) and (bottom) TMS/TIMS (approximately 12-m resolution and $24 \times 6 \mathrm{~km}^{2}$ extent) datasets.

mation of emissivity obtained from the fractional vegetation cover as follows:

$$
\bar{\varepsilon}=\varepsilon_{\text {veg }} \cdot \mathrm{Fr}+\varepsilon_{\text {soil }} \cdot(1-\mathrm{Fr}),
$$

with $\varepsilon_{\text {veg }}=0.985$ assigned as a representative value for vegetation emissivity and $\varepsilon_{\text {soil }}=0.955$ for bare soil.

An issue of primary importance with the comparison of radiometric temperatures and fluxes derived from these temperatures was the fact that the times of data collection by the aircraft and satellites were not concurrent. The airborne data was collected at approximately 1000 LST, while the AVHRR data was from an afternoon overpass at approximately 0200 LST. To bring the data to the same time, a linear regression was used to relate the TIMS temperature to 0200 LST so that it can be used to calculate fluxes that are comparable with the AVHRR calculated fluxes. This methodology is similar to that expounded by Lagouarde (1993) to enhance the utility of the thermal channel of thematic mapper (TM) data, which a is similarly early morning mea- surement. This technique is described in more detail in Brunsell and Gillies (2002b).

\section{b. Derivation of surface fluxes}

Surface fluxes of latent heat (LE), sensible heat (H), and net radiation $(\mathrm{Rn})$ were only derived for the TMS/ TIMS and the AVHRR datasets. The TM data was not used because the satellite overpass was approximately 2 weeks after the collection of the airborne data and thus the fluxes would not be directly comparable. The analysis was conducted at two resolutions and extents; $12 \mathrm{~m}$ for the TMS Fr and TIMS radiometric temperature airborne data and $1000 \mathrm{~m}$ for the AVHRR data.

A Soil-Vegetation-Atmosphere Transfer (SVAT) model was used to derive the energy fluxes as a function of the remotely sensed vegetation and radiometric temperature. The SVAT model is essentially a one-dimensional, two-source model with a boundary layer component. It is one-dimensional in the sense that the de- 

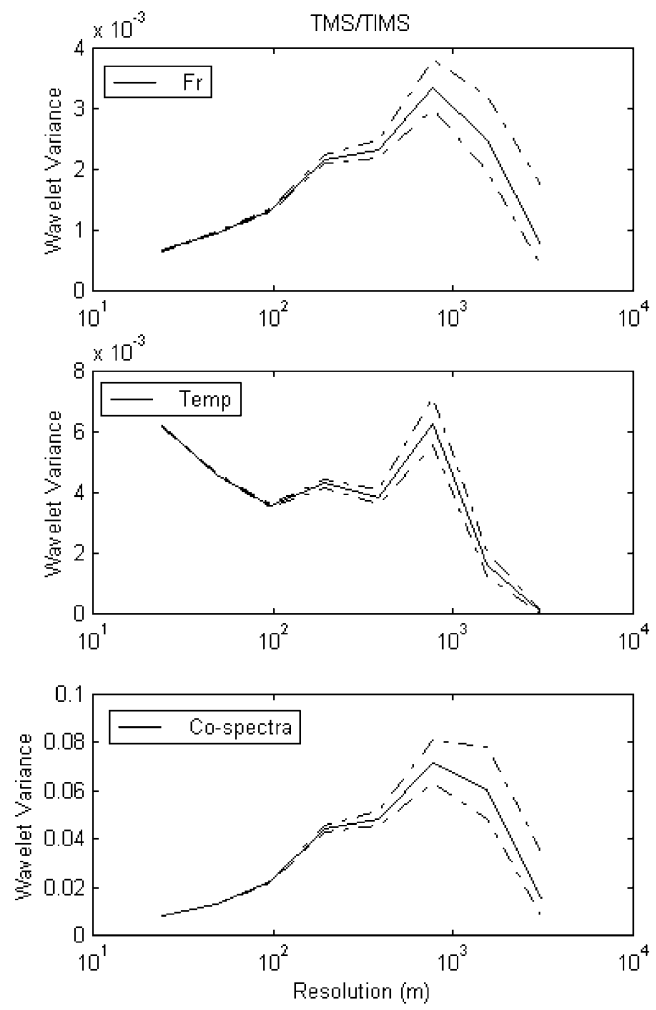
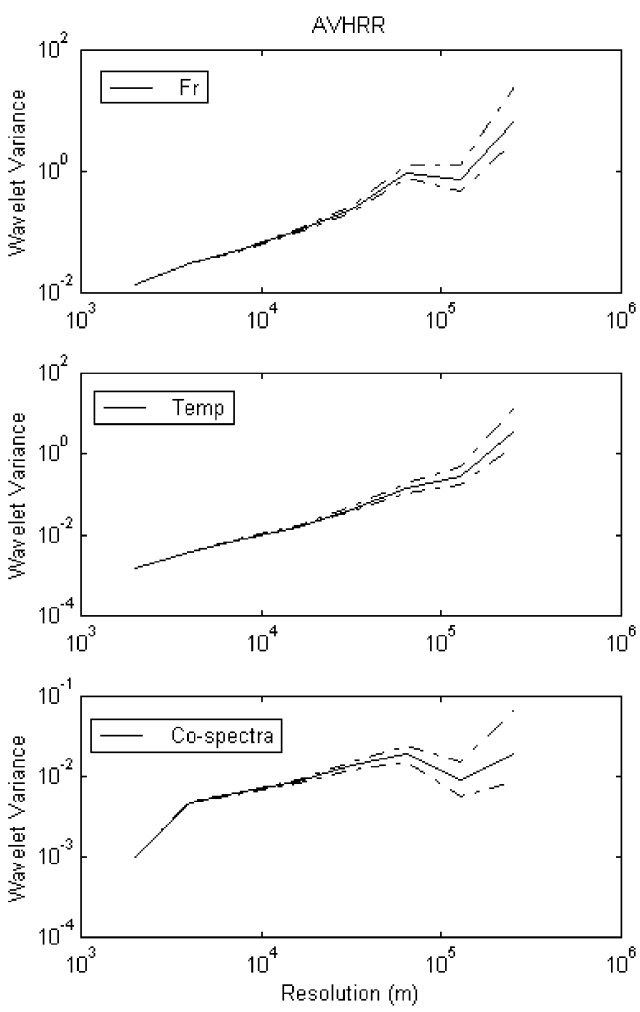

FIG. 2. Wavelet spectra for (left column) TMS and (right column) AVHRR (top) Fr, (middle) temperature, and (lower) Fr-temperature cospectra. Note the logarithmic scale on the $y$ axis of the AVHRR spectra; confidence intervals are $90 \%$ confidence limits

rived fluxes are based on the assumption that large-scale horizontal variability is not important with respect to vertical fluxes. There is a slight incorporation of largerscale processes due to the initialization with a local radiosonde that does allow for some large-scale advective processes. However, it is assumed that the effect of the surface horizontal variability is minor [in the terminology of Raupach and Finnigan (1995), the model assumes microscale variability]. The SVAT model is a two-source model in that each pixel-scale flux is modeled as a linear average of the soil and vegetation fluxes, which are computed separately. The two components (soil and vegetation) are independent of one another, except that they both alter the conditions of the lower atmosphere, which is then used to determine the flux at the following time step. For a further description of the model see Carlson (1986), Lynn and Carlson (1990), Carlson and Boland (1996), and Gillies et al. (1997).

An in-depth discussion of the model and the resultant fluxes, as well as comparison with surface measurements, is presented in Brunsell and Gillies (2002b). However, for the sake of completeness, a short description is provided here. The SVAT model is initialized with a morning radiosonde profile and evolves the boundary layer over the course of the day. For a given value of surface vegetation cover and near-surface soil moisture (Gillies et al. 1997), the model evolves the surface energy fluxes using the air fields and radiation regime for that day and location.

The triangle method relies on the shape of the remotely sensed data in the Fr-radiometric temperature space. The data is assumed to contain the full range of vegetation cover and near-surface soil moisture availability (Gillies et al. 1997). The four corners of the trapezoid shape of the $\mathrm{Fr}-T_{\text {rad }}$ space are assumed to represent four cases: (i) completely vegetated, completely saturated soil; (ii) completely vegetated, completely dry soil; (iii) no vegetation, saturated soil; and (iv) no vegetation, dry soil. Following calibration, the model is iterated over all possible vegetation covers and soil moisture availabilities, and the output fluxes are used to calculate regression equations to relate surface energy fluxes and the soil moisture availability with modeled radiometric temperature and input fractional vegetation using

$$
F_{x}=\sum_{i=0}^{3} \sum_{j=0}^{3} a_{i, j} T^{i} \operatorname{Fr}^{j}
$$

where $F_{x}$ is the predicted value of the flux, $T$ is the pixel radiometric temperature, $\mathrm{Fr}$ is the pixel fractional vegetation, and $a_{i, j}$ are the regression coefficients. Equation (4) is then applied to the remotely sensed data using the 

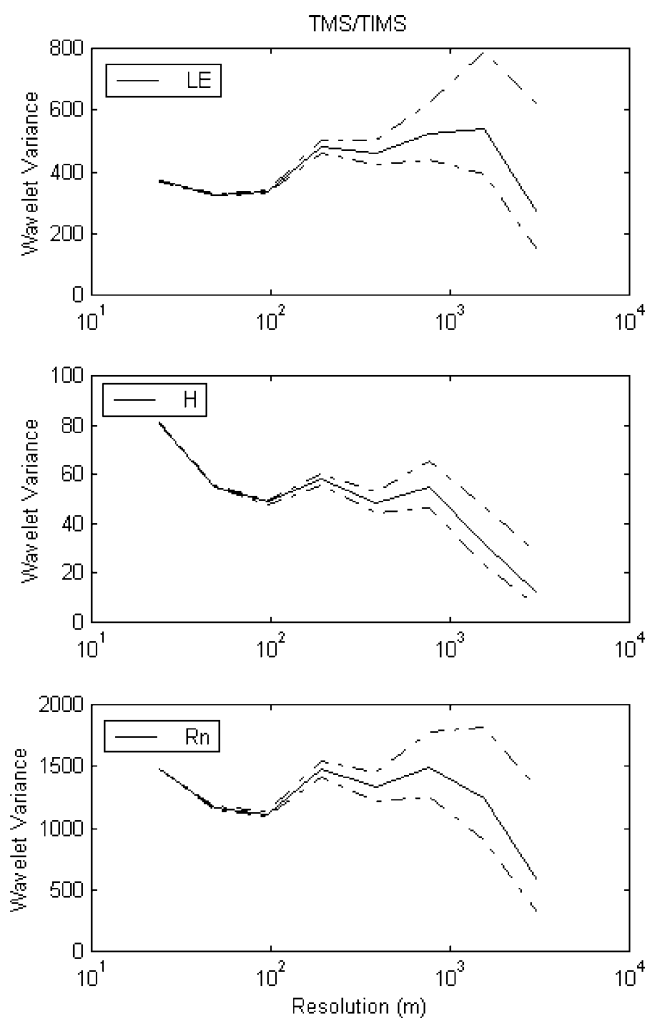
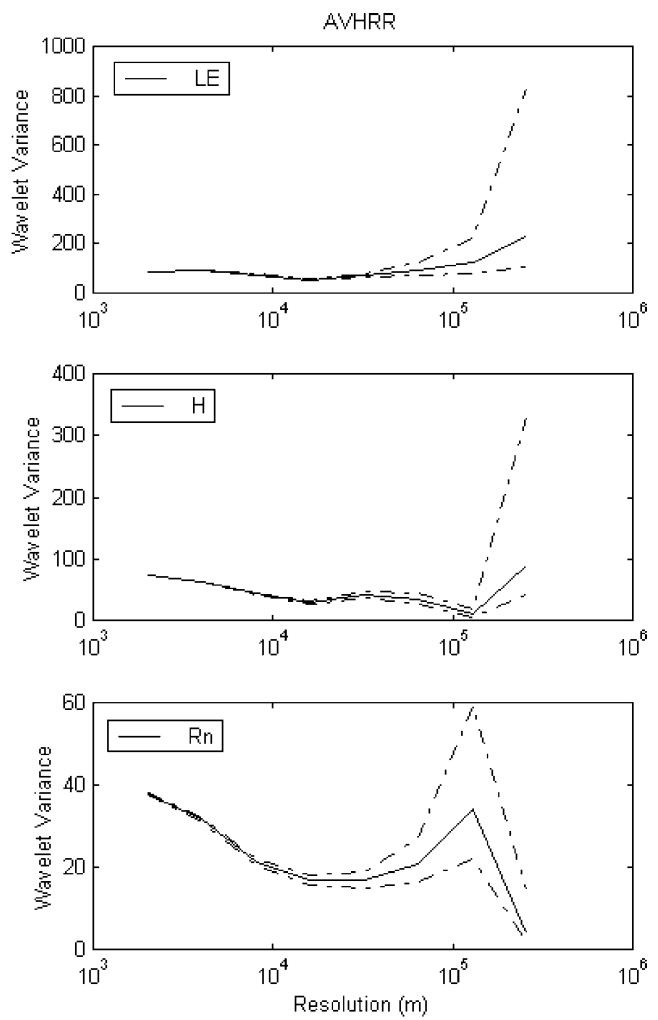

FIG. 3. Length scales for SVAT output based on (left column) TMS Fr and TIMS temperature and (right column) AVHRR (top) LE, (middle) $H$ and (bottom) Rn. Confidence intervals are at the $90 \%$ level.

actual radiometric temperature and fractional vegetation to estimate pixel-scale fluxes from the data.

\section{c. Wavelet spectra}

To understand the scaling behavior of the fractional vegetation and radiometric temperature data, a multiresolution wavelet analysis was conducted. Wavelet analysis is benefical over more traditional forms of analysis for the localization in both the spatial and scale domains. This localization provides a robustness to nonstationarities in the data that is not possible with other types of analysis (e.g., semivariograms, etc.).

The two-dimensional discrete wavelet transform using the Daubechies 2 wavelet was used to compute spectra. The Daubechies 2 wavelet is smoother than the usually applied Haar wavelet and thus will be able to detect more subtle changes than the Haar wavelet at each scale. The Haar wavelet can detect sharp transitions, but more gradual transitions actually occuring at the same scale may be computed as occuring at a coarser scale. Therefore, the Daubechies 2 wavelet was chosen as being beneficial over the Haar. The wavelet spectra were used to identify the nature of any scaling within the observed signal. The dominant length scale is defined as the scale at which the wavelet variance is a maximum (Kumar and Foufoula-Georgiou 1997). The dominant length scale is defined without respect to the direction of ori- entation; thus the detail coefficients in both directions (i.e., the diagonal coefficients) of the wavelet transform were used in the computation of the spectra (see Percival 1995 and Kumar and Foufoula-Georgiou 1993):

$$
\hat{v}_{\lambda}=\sum_{x}\left|D_{x, \lambda}\right|^{2}
$$

where $D$ is the wavelet coefficient from a discrete wavelet transform at location $x$ (a vector in two-dimensional space), resolution is $\lambda$, and $\hat{v}$ is the variance at that resolution. Confidence intervals are computed according to Percival (1995).

\section{Results}

a. Horizontal length scales of surface fluxes, radiometric temperature, and fractional vegetation

The first issue examined was the variation across scale of the remotely sensed temperature, vegetation, and derived energy balance fluxes. This analysis was conducted for two datasets for which radiometric temperature data was available on 2 July 1997: (i) the TMS/ TIMS data at $12-\mathrm{m}$ resolution for the extent of approximately $24 \times 6 \mathrm{~km}^{2}$ and (ii) the AVHRR data at $1000-\mathrm{m}$ resolution for a much larger extent of $512 \times 512 \mathrm{~km}^{2}$. These extents are shown in Fig. 1. The fluxes of latent heat, sensible heat, and net radiation were computed for the AVHRR and TMS/TIMS as described in section $2 \mathrm{~b}$. 

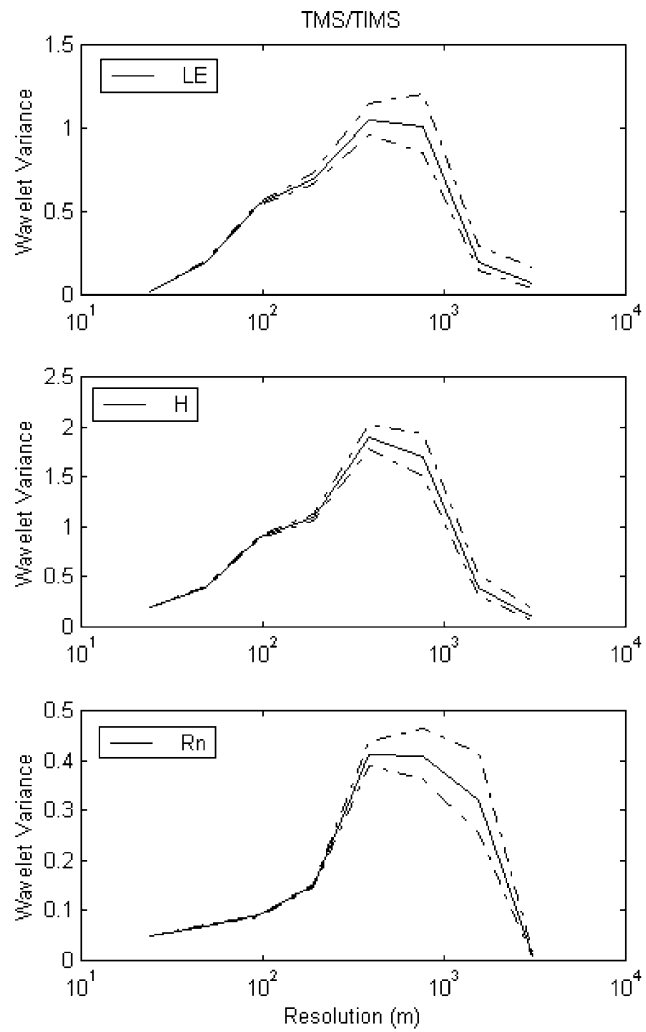
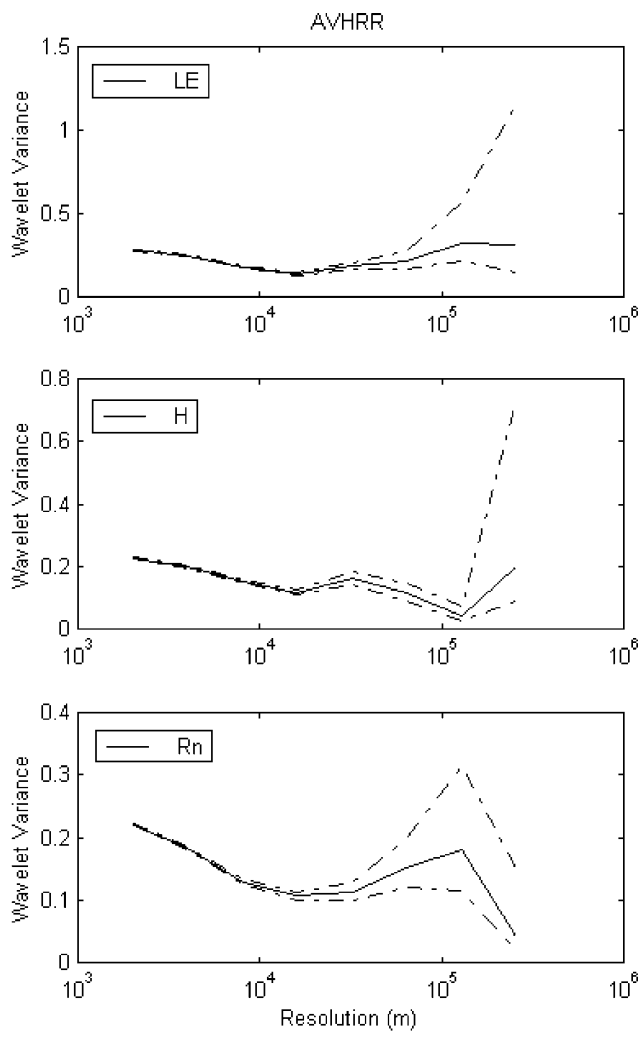

FIG. 4. Wavelet cospectra for (left column) TMS/TIMS and (right column) AVHRR fluxes and Fr (top) LE, (middle) $H$, and (bottom) Rn, with $90 \%$ confidence intervals.

The spectra for TMS fractional vegetation are presented in Fig. 2 along with the TIMS temperature spectra. Both datasets show a length scale on the order of $800 \mathrm{~m}$, while the temperature also shows a peak at a much smaller resolution of $24 \mathrm{~m}$. The wavelet cospectra between the temperature and $\mathrm{Fr}$ are presented in Fig. 2 with a peak at $800 \mathrm{~m}$ and extending into the 1600-m region.

The AVHRR temperature, fractional vegetation, and cospectra are also presented in Fig. 2. The fractional vegetation and temperature spectra show near log-log linearity in their spectra, both dominated by larger-scale variability. The cospectra are dominated by scales larger than $64000 \mathrm{~m}$.

The analysis was also conducted for the derived energy fluxes. These spectra are shown in Fig. 3. The latent heat field shows relatively constant influence above about $200 \mathrm{~m}$, while the sensible heat flux is dominated by smaller-scale variability on the order of $24 \mathrm{~m}$. The net radiation has characteristics of both-a peak in the 24-m region, as well as the larger-scale influence of 200-2000 m.

Spectra for the derived fluxes from the AVHRR data are also shown in Fig. 3. The latent heat appears to follow the temperature and fractional vegetation data and is largely dominated by larger scales. The sensible heat flux shows some contribution from both the largest and smallest scales. The net radiation shows the same with peaks in the 2000- and $128000-\mathrm{m}$ scales.

\section{b. Interaction between length scales of vegetation, temperature, and surface energy fluxes}

One of the dominant interests in understanding the scaling in land-atmosphere interactions is how the scaling behavior of the controlling variables is reflected in the scaling of the derived fluxes. In order to examine this issue wavelet cospectra were calculated between the fluxes and the radiometric temperature and fractional vegetation. This was done because these fields represent the input fields for which it is relatively easy to ascertain spatial variability with remote sensing, and which the triangle method uses to estimate the flux terms.

Figure 4 presents the wavelet cospectra between $\mathrm{Fr}$ and the fluxes for both the TMS/TIMS (left column) and the AVHRR (right column) estimates. Figure 5 presents the same for the cospectra between radiometric temperature and the fluxes.

The same results are observed in all of the plots for the TMS/TIMS cospectra: a peak on the order of 400 $800 \mathrm{~m}$. The $800-\mathrm{m}$ peak is still observed, but almost of more importance is the appearance of the $400-\mathrm{m}$ peak, since this is not a dominant scale in any of the individual fluxes or input fields. Other interesting results are the 

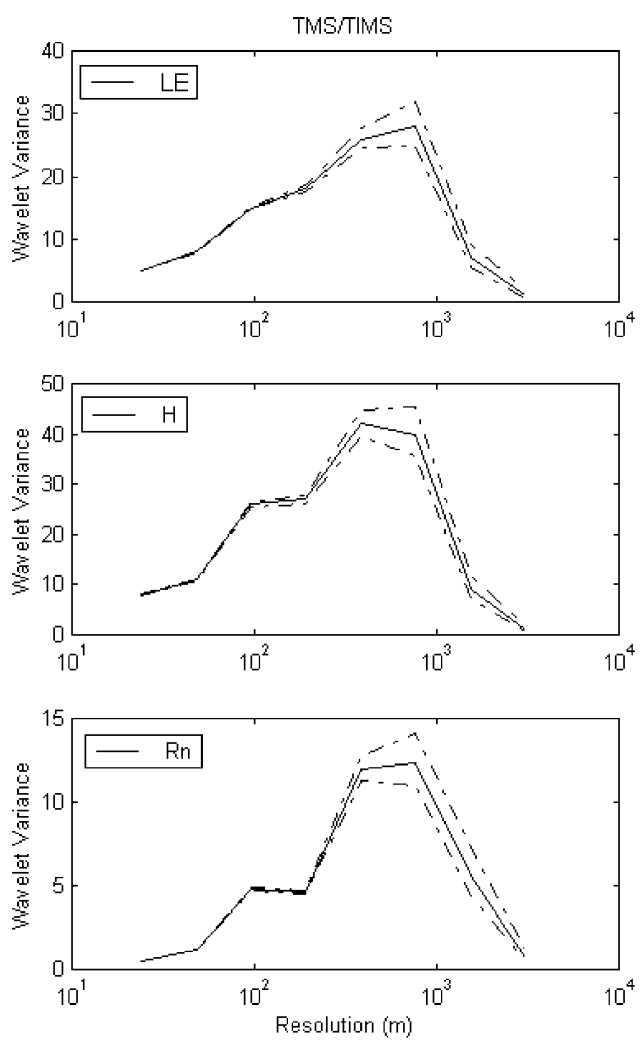
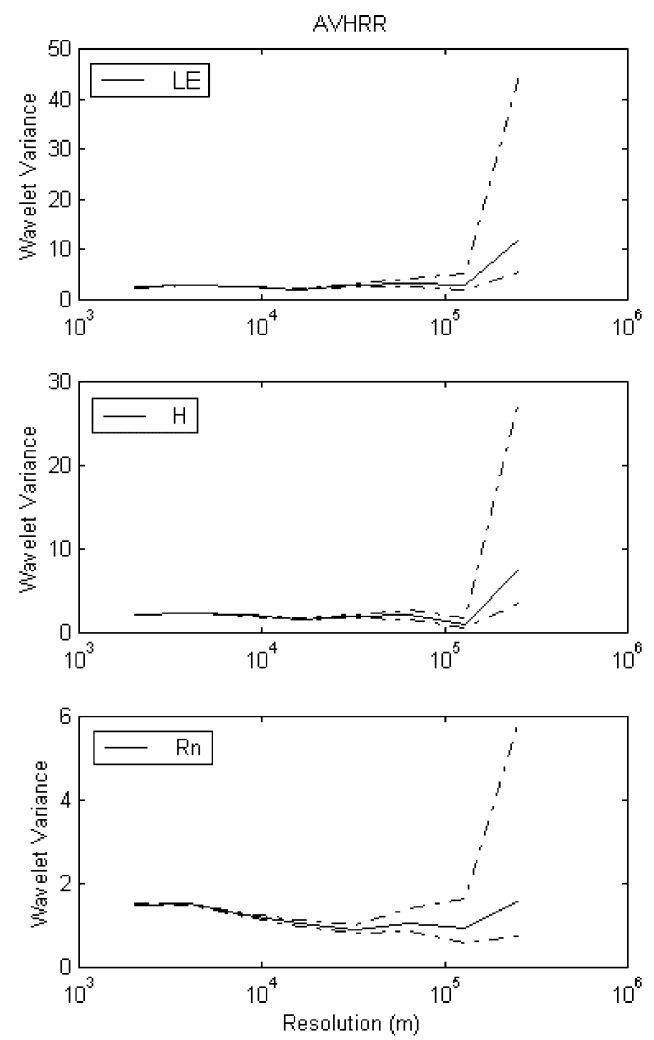

FIG. 5. Wavelet cospectra for (left column) TMS/TIMS fluxes and (right column) AVHRR and temperature (top) evaporation, (middle) sensible heat, and (bottom) net radiation; 90\% confidence limits are also shown.

disappearance of all the small-scale variation that was present in the temperature and the flux fields. It is especially interesting in the cospectra between Fr and LE and Fr and $\mathrm{Rn}$, since in the flux spectra this small-scale variability was presumably due to the variation in the temperature field.

The cospectra for the AVHRR fractional vegetation are presented in Fig. 4. The smallest resolvable scale $(2000 \mathrm{~m})$ is again one of the dominant scales for cospectra between the fluxes and the fractional vegetation, with the other dominant influence being the larger scales. This is of interest, especially for the latent heat field, since there was relatively no small-scale variability observed within the LE spectra. The net radiation-Fr cospectra also shows the relative importance of the 128 000-m scale, which is of less importance in the LE and $H$ fields.

The AVHRR flux-temperature cospectra is shown in Fig. 5. Each of these fields shows the dominant scale as the largest with relatively constant influence over the other scales, with the possible exception of the net radiation-temperature cospectra having some influence from the smallest scales.

\section{Discussion and conclusions}

This paper examines how the variability in dominant remotely sensed fields is translated into variability in the derived surface energy balance fields. It is well known that many aspects of land-atmosphere interactions do not scale linearly, in that spatial averages of input data do not yield the true spatial average of the flux. However, there have been few opportunities to assess how the spatial variability of these fields observed at different scales (i.e., pixel resolutions) translates into spatial variability in modeled fluxes.

Of course, the use of the "triangle method" (Gillies et al. 1997) carries with it some assumptions that may or may not be true, but Brunsell and Gillies (2002b) have shown reasonable agreement between measured fluxes and estimates derived from the remote sensing data. The most obvious assumption is that the energy fluxes of the vegetation and bare soil can be computed separately and only interact through the mutual alteration of the lower atmosphere for the next time step. However, this assumption is often made in the application of remote sensing data to estimate surface energy fluxes and may not be as severe as it first sounds.

The analysis of the variation in length scales between input data and derived fluxes revealed that the highresolution remote sensing data available from the TMS/ TIMS flights is largely dominated by the 400-800-m scales, which corresponds closely to the average size of the agricultural fields in the El Reno area (approximately $800 \mathrm{~m}$ ). The fact that the airborne-derived sensible heat 
flux exhibits small-scale variability, as does the temperature field, might lead one to suspect that the scaling nature of the sensible heat flux is determined by the temperature field. However, the cospectral analysis does not show any small-scale variability like that seen in the temperature, sensible heat, or net radiation fields.

One possible explanation for this is that the variability observed within the temperature data does have a peak at the $800-\mathrm{m}$ scale but is relatively constant up to that scale. This implies that although the $800-\mathrm{m}$ peak is dominant within the resultant cospectra, this influence is most easily caught within the determination of the fluxes. This also explains the presence of the 400-m length scale observed in the cospectra. This scale is not the overall dominant scale in any field; however, it is significant, which becomes more obvious in the cospectra.

The AVHRR data show a different trend. Since the AVHRR has an initial pixel size of $1000 \mathrm{~m}$, the largerscale features dominate because the resolution is too coarse to observe the small-scale (field to field) variability that dominated the temperature field in the highresolution data. At the smaller scale, though, variability in albedo, which can vary at the subfield scale and is most easily represented within the fractional vegetation data (since it is determined as a function of the reflectance in the red and near-infrared data), is dominant over the small-scale variability in solar radiation and the resultant temperature fields. This results in slightly higher small-scale peaks in the cospectra between the fluxes and the fractional vegeation $(2000 \mathrm{~m})$ relative to the temperature-flux cospectra, which exhibit largescale variability almost exclusively.

The implication of this study for land-atmosphere interactions is that not only are the fluxes not determined by the linear average of the controlling parameters, but the length scales of the variability are also not maintained. The small-scale variability in the temperature field dominates the flux fields. The dominant scale in the fractional vegetation as well as the cospectra between the temperature and the vegetation fields is reduced in importance within the flux fields. This, however, is not true of the cospectra between the fluxes and the remotely sensed data, where the dominant scales are 400-800 $\mathrm{m}$. This implies that knowing the dominant scales of the remotely sensed data may not be enough to know the spatial variability of the surface energy fluxes. More research is necessary to determine the generality of these results.

Acknowledgments. The authors wish to thank Andy French and Tom Schmugge of the USDA-ARS, for assistance with the TMS/TIMS data. Funding was provided through a grant from NASA Cooperative Agree- ment NCC8-162 in support of the Global Energy and Water Cycle Experiment (GEWEX) Continental-Scale International Project (GCIP) and Utah Agricultural Experiment Station, Utah State University, Logan, Utah.

\section{REFERENCES}

Brunsell, N. A., and R. R. Gillies, 2002a: Incorporation of surface emissivity into a thermal atmospheric correction. Photogramm. Eng. Remote Sens., 68, 1263-1269.

$\ldots$, and $-2002 \mathrm{~b}$ : Scale issues in land-atmosphere interactions: A review, with implications for remote sensing of the surface energy balance. Agric. For. Meteor., 117, 203-221.

Carlson, T., 1986: Regional scale estimates of soil moisture availability and thermal inertia using remote thermal measurements. Remote Sens. Rev., 1, 197-247.

__ , and F. E. Boland, 1996: Will a doubling of atmospheric carbon dioxide concentration lead to an increase or a decrease in water consumption by crops? Ecol. Modell., 88, 241-246.

_- and D. Ripley, 1997: On the relation between NDVI, fractional vegetation cover, and leaf area index. Remote Sens. Environ., 62, 241-252.

Choudhury, B., N. Ahmen, S. Idso, R. Reginato, and C. Daughtry, 1994: Relations between evaporation coefficients and vegetation indexes studied by model simulations. Remote Sens. Environ., 50, $1-17$.

Diak, G. R., 1990: Evaluation of heat flux, moisture flux and aerodynamic roughness at the land surface from knowledge of the PBL height and satellite-derived skin temperatures. Agric. For. Meteor., 52, 181-198.

French, A. N., T. J. Schmugge, and W. P. Kustas, 2000: Estimating surface fluxes over the SGP site with remotely sensed data. Phys. Chem. Earth, B25, 167-172.

Gillies, R., T. Carlson, J. Cui, W. Kustas, and K. Humes, 1997: A verification of the "triangle" method for obtaining surface soil water content and energy fluxes from remote measurements of the normalized difference vegetation index (NDVI) and surface radiant temperature. Int. J. Remote Sens., 18, 3145-3166.

Kniezys, F. X., and Coauthors, 1996: The MODTRAN 2/3 Report and LOWTRAN 7 Model. Phillips Laboratory, Hanscom AFB Tech. Rep. F19628-91-C-0132, 137 pp.

Kumar, P., and E. Foufoula-Georgiou, 1993: A multicomponent decomposition of spatial rainfall fields. 1. Segregation of largeand small-scale features using wavelet transforms. Water Resour. Res., 29, 2515-2532.

__ and - , 1997: Wavelet analysis for geophysical applications. Rev. Geophys., 35, 385-412.

Kustas, W., T. Jackson, A. French, and J. MacPherson, 2001: Verification of patch and regional scale energy balance estimates derived from microwave and optical remote sensing during SGP97. J. Hydrometeor., 2, 254-273.

Lagouarde, J. P., 1993: Using mid-morning surface temperature in the framework of future TIR satellite systems. Proc. Workshop on Thermal Remote Sensing of the Energy and Water Balance over Vegetation in Conjunction with Other Sensors, La Londe les Maures, France, PSU, CEPT, and CEMACREF-ENGREF (Remote Sensing Lab), 227-232.

Lynn, B., and T. N. Carlson, 1990: A stomatal resistance model illustrating plant vs. external control of transpiration. Agric. For. Meteor., 52, 5-43.

Percival, D., 1995: On estimation of the wavelet variance. Biometrika, 82, 619-631.

Raupach, M., and J. Finnigan, 1995: Scale issues in boundary-layer meteorology: Surface energy balances in heteorgeneous terrain. Hydrol. Processes, 9, 589-612. 